\title{
Mit den Beschützern der Diebe durch Berlin
}

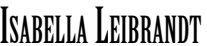

Universidad de Navarra

«Heute ist fast jeder Krimi mehr als ein Krimi» behaupten die Kenner dieser Gattung. Im Folgenden möchte ich den Krimi «Beschützer der Diebe» von Andreas Steinhöfel vorstellen, der bereits zu den besten Beispielen dieser Gattung zählt und nicht nur eine spannende Lektüre innerhalb der Jugendliteratur ist, sondern an dieser Stelle unter didaktischen Gesichtspunkten für Deutschlerner jeden Alters schmackhaft gemacht werden will.

Zunächst einmal sollen kurz einige fundamentale Fragestellungen umrissen werden: Was zeichnet die Textsorte des Kinderkrimis im Allgemeinen aus und welche Inhalte machen sie auch für erwachsene Lerner zu einer lesenswerten Lektüre? Bislang steht der Kinderkrimi wie die gesamte Kinder- und Jugendliteratur im Schatten der 'großen' Literatur und gilt häufig als geringgeschätze Trivialliteratur. Aber wer verdankt nicht einige seiner spannendsten und unterhaltsamsten Lebensmomente den großen Kinderdetektiven wie Emil, Kalle Blomquist oder dem JuniorDeketivteam «die Drei???»

Wer diese Figuren jedoch nur verschwommen in der Erinnerung behalten hat, der möge sein Gedächtnis auffrischen: Die drei Fragezeichen sind drei Jungen aus Rocky Beach in Kalifornien: Justus, Bob und Peter. Was einst als Zeitvertreib begann, hat sich längst zu einer ernsthaften Nebenbeschäftigung für freie Stunden und Schulferien entwickelt: Das Junior-Detektivteam «die drei
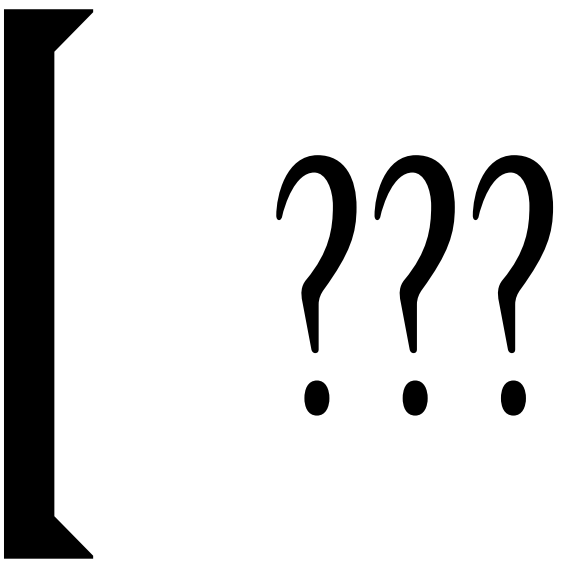

mente die Hauptattraktivität jeder Krimilektüre. Damit ist das Rätsel oder die 'Whodunit'-Frage das Hauptcharakteristikum jeder klassischen Detektivgeschichte: der Leser verfolgt auf den Spuren der genialen Detektive die Auflösung eines Verbrechens, wird somit aktiver Teilhaber an der Spurenverfolgung, sein Verstand und die Cleverness werden herausgefordert während er zusammen und scheinbar um die Wette mit dem Detektiv das Rätsel lösen will. In «Beschützer der Diebe» ist es zunächst ein gefundener Zettel mit den Buchstaben und Zahlen KEM 5018, der den Protagonsiten und dem Leser Kopfzerbrechen bereitet. Die Hauptfigur des Kinderdetektivs befindet sich jedoch selten allein auf des Rätsels Spur, sondern berät sich mit unerlässlichen Partnern (Freunden), die ihn häufig aus einer verzweifelten Notlage oder sogar lebensgefährlichen Situation erlösen und damit wichtige Eigenschaften aus der kindlichen Lebenswelt wie Vertrauen, Freundschaft, Mut und die Herausforderungen des Lebens verkörpern. Der Reiz für den (jugendlichen) Leser liegt nicht nur darin, dass er im Wettstreit mit dem Helden mitfiebert und miträtselt, sondern aus der kindlichen Perspektive gesehen, er sich mit der genialen Detektivfigur identifiziert und selbst für einige Lebensaugenblicke zum Helden wird. Am erfolgreichsten ist die Leser-Detektivfigursymbiose sicherlich, wenn Schlussfolgerungen und Gedankengänge der Hauptfigur zu den eige???» hat schon oft die Aufklärung geheimnisvoller Fälle geliefert. Als Zentrale haben sich die drei einen ausrangierten Wohnwagen eingerichtet (selbstverständlich mit allen notwendigen Apparaturen, die den Jungs modernste Ermittlungstechniken ermöglichen). Jeder Fall erfordert vorwiegend Köpfchen, Ausdauer und Mut.

Emil darf zum erstenmal allein nach Berlin fahren. Seine Großmutter und die Kusine Pony Hütchen erwarten ihn am Blumenstand im Bahnhof Friedrichstraße. Aber Emil kommt nicht, auch nicht mit dem nächsten Zug. Während die Großmutter und Pony Hütchen noch überlegen, was sie tun sollen, hat sich Emil schon in eine aufregende Verfolgungsjagd gestürzt. Quer durch die große fremde Stadt, immer hinter dem Dieb her, der ihm im Zug sein ganzes Geld gestohlen hat. Zum Glück bekommt Emil bald Unterstützung von Gustav mit der Hupe und seinen Jungs.

Sicherlich bilden zunächst die Abenteuer- und Spannungsele- nen werden oder sogar durch den Leser vorweggenommen werden. Wie in der gesamten Kinder- und Juegndliteratur so besteht auch im Krimi ein weiteres Merkmal darin, dass die Darstellung der Kinderwelt häufig der Welt der Erwachsenen gegenübergestellt wird, mit dieser sogar in diversen Problemsituationen kollidiert, die Heranwachsenden nach eigenen Lösungen suchen lässt, ihnen Freiraum zum Handeln verschafft, sie im Reifeprozess begleitet und lehrt selbständig zu werden.

\section{Kinder- und Jugendliteratur im Daf-Unterricht}

Im Hinblick auf die Verwendung von KJL im Daf-Unterricht besteht inzwischen die einheitliche Meinung, dass sie nicht nur für Kinder sondern auch für (junge) Erwachsene einen unersetzbaren Themenreichtum bei der Kulturvermittlung gewährleistet und die Grundlage für diverse interkulturelle Vergleiche 
bildet. Aus der vermittelnden Perspektive betrachtet und hinsichtlich einer Integration dieser Textsorte in den Fremdsprachenunterricht bietet der besondere Adressatenbezug und das pädagogisch-didaktische Potential der KJL eigene Wege der Annäherung an einen literarischen Text, an das interkulturelle Lernen sowie die Landeskunde. Die Tatsache, dass realistische und phantastische Geschichten wie in allen fiktionalen Textsorten gemischt werden, spielt eine große Rolle. Im Kinder- und Jugendbuch wird das 'Realistische' der 'realistischen Kindergeschichte' darin gesehen, dass diese die Kinder und deren Umwelt möglichst wirklichkeitsgetreu zu treffen versucht. Der Begriff der 'realistischen Kindergeschichte' wird infolgedessen häufig synonym mit dem Begirff der 'Umweltgeschichte' (problemorientiertes Jugendbuch) benutzt, deren Rezeption Freude und Spaß macht, aber gleichzeitig zum Nachdenken anregt. Weitgehend einig ist man sich heute darüber, dass die 'phantastische' Literatur, ebenso wie die sogennante 'realistische' Literatur, Erkenntnisfunktion besitzt. Im Rahmen einer umfassenden Funktionsbestimmung werden deshalb folgende Aspekte herausgestellt: die Erweiterung des Wirklichkeitsverständnisses, die Aktivierung der nicht-nur-rationalen Erfahrungen sowie die Möglichkeit, konkrete und direkte Anstöße zur Wirklichkeitsveränderung zu liefern sowie Wissen, Informationen und pädagogische Intentionen zu transportieren. Die Idee, KJL im DaF-Unterricht einzusetzen ist naheliegend, vor allem wenn man zunächst einmal sprachliche Aspekte bei der Auswahl in Betracht zieht. Darüberhinaus kann die KJL eine Brücke zwischen den fortgeschrittenen kognitiven Fähigkeiten der Lernenden und deren (geringer) Sprachfähigkeit bilden, doch sie birgt auch noch andere Vorteile. So spricht Literatur im allgemeinen den Leser auf einer emotionalen Ebene an, führt zu einer emotionalen Beteiligung und animiert zum Nachdenken. Neben der reinen Sprachvermittlung bildet auch die Landeskunde einen Schwerpunkt des DaF-Unterrichts, wobei deren Vermittlung durch die KJL erleichtert werden kann. So erhalten die Lernenden durch den Einsatz von KJL nicht nur einen Einblick in unterschiedliche Realitäten, sondern je nach Perspektiveneinstellung erfährt der Fremdsprachenlerner vielmehr, welche Probleme es in Deutschland gibt, worüber sich die Menschen sorgen, wovon man spricht und träumt, und welche sozialen und politischen Verhältnisse die Deutschen beeinflussen. Durch die unterschiedlichen Perspektiven können also Rückschlüsse auf das Verhalten der Menschen im deutschsprachigen Kulturraum gezogen werden. Das Projekt « 105 Kinder- und Jugendbücher in Deutsch als Fremdsprache-Unterricht» (http://web.uni-frankfurt.de/fb10/jubufo/studprojekt/kjldaf/main.html) stellt dazu eine umfangreiche Fundgrube zur Verfügung.

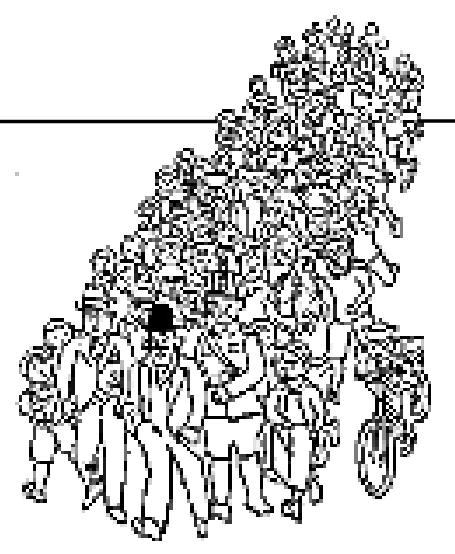

\section{Der Autor und die Beschützer der Diebe}

Andreas Steinhöfel wird heutzutage zu den besten Kinder- und Jugendbuchautoren Deutschlands gerechnet. Eine Vielfalt an aktuellen Themen kommt in seinen Kinder- und Jugenbüchern zur Sprache: Bewältigungsanforderungen in der spießigen Enge der Kleinstadt, Beziehungskonflikte in der Familie und Partnerschaft und besonders Steinhöfels Umgang mit dem Thema Homosexualität - das er auch in seinem packenden Kinder-Krimi «Beschützer der Diebe» streift - scheint typisch für einen neuen Ton im deutschen Kinderbuch zu sein. Damit zeigt er, dass Jugendliteratur mehr sein kann als reine Unterhaltungsliteratur, denn mit großer Selbstverständlichkeit wird über Scheidungen, Geldsorgen, Arbeitslosigkeit und Liebeskummer, den Hang zum Kaufhausdiebstahl, Vorurteile, das Großstadtleben und die Kulturpolitik in Bezug auf Berlin berichtet. Dabei geht es nicht um eine reine didaktische Vermittlung von "Problemthemen», Andreas Steinhöfel und andere zeitgenösische Autoren schreiben so, weil das Leben so ist. Dabei faszinieren den Autor vor allem Unterwelten jeder Art, diejenigen von Städten und von Seelen insbesondere und die Frage, ob es nicht neben und unter den neonerleuchteten Bahnhöfen und schwarzen Tunneln der U-Bahn noch eine andere Welt geben muss, in die gelangen kann, wer genau genug hinschaut. In «Beschützer der Diebe» sind es die geheimen Gänge unter dem Pergamon-Museum, durch die sich die Helden der Geschichte schlagen müssen, um einen besonderen Schatz zu retten: das berühmte Tor von Milet, das skrupellose Kunsträuber gegen eine Attrappe austauschen wollen. Ein kühner Plan, besonders angesichts der Tatsache, dass dieses Ausstellungsstück etwa 20 Meter hoch und 30 Meter breit ist. Schauplatz ist - wie in Erich Kästners «Emil und die Detektive» - die Stadt Berlin. Zwei Mädchen und ein Junge geraten in immer gefährlichere Situationen bei der Klärung eines Verbrechens, in das sie durch den puren $\mathrm{Zu}$ fall verwickelt werden. Doch die jugendlichen Helden können sich auch in kritischen Augenblicken aufeinander verlassen und verhindern in letzter Minute den Kunstdiebstahl. Alles beginnt mit einem scheinbar harmlosen Spiel während der Sommerferien: jeder der drei sucht sich eine Person aus, die in der Stadt herumläuft und verfolgt ihn oder sie solange bis er über die verfolgten Personen so viel wie möglich herausbekommen hat. Auf diese Weise wird Guddie, die noch nicht lange in Berlin wohnt Zeugin, wie vor ihren Augen ein Mann entführt wird, als er aus dem Museum kommt. Da die Polizei kein Interesse zeigt, versucht sie gemeinsam mit Dagmar und Olaf den Fall aufzuklären. Als einziges Beweismittel besitzen sie einen kleinen Notizzettel. Ihre nervenaufreibenden Nachforschungen führen sie quer durch Berlin, immer wieder an unbekannte Orte. Neben 
der abenteuerlichen Detekivgeschichte über einen raffinierten Kunstraub geht es dem Autor aber um die spezifischen Biografien der drei Protagonisten. Als Verfasser von erzählender Literatur für junge Leser stellt Andreas Steinhöfel drei Jugendliche ins Zentrum seiner Geschichte und als handlungstragende Protagonisten, sympathisiert mit den Kindern, ergreift für sie, wie Erich Kästner oder Astrid Lindgren, Partei und zeigt, dass auch Kinder bereits neben ihren eigenen Schwierigkeiten beim Erwachsenwerden mit Erwachsenenproblemen zum Beispiel von Alleinerziehenden Stärke zeigen müssen. Neu dabei ist die Thematisierung der Lebenssituation von Homosexuellen, des Fotografen Bernd Wörlitzer und seinem Freund Stefan, die die Kinder väterlich-verständnisvoll unterstützen. Steinhöfel offenbart damit ein Interesse am Anderen, am Befremdlichen, an Charakteren, deren Lebensstil nicht der gesellschaftlichen Norm entspricht und unterstellt seinen Protagonisten Möglichkeiten der Entscheidung mit Vorurteilen zurecht zu kommen, gibt ihnen Perspektiven, sich zu entwickeln und zu verändern.

Damit sind Guddie, Dags und Olaf real wirkende Jugendliche mit eigenen Problemen und Spinnereien und keine stereotypen Hobby-Detektive wie die drei ???. Dagmar fällt durch ihre Selbständigkeit und Extravaganz ganz aus der mädchenhaften Klischeerolle, führt ständig ihre Ratte Romeo, mit der sie Experimente veranstaltet, mit sich, versucht sie zu dressieren, interessiert sich dank ihres Vaters, einem Professor an einem Krebsforschungsinstitut für die neusten wissenschaftlichen Erkenntnisse und ist das 'Superhirn' der drei, hat aber trotz einer intakten Familie auch genug mit sich selbst zu tun:

«Dags ließ Romeo in die geräumige, von ihr selbst angenähte und mit einem Reißverschluss versehene Innentasche ihrer Jeansjacke gleiten und musterte sich in dem Spiegl. Ein blaues und ein braunes Auge blickten zurück - die einzig wirklich auffälligen Merkmale in ihrem runden Gesicht, das von einem Wust widerspenstiger rotbrauner Locken eingerahmt wurde. Die unterschiedliche Farbe ihrer Augen war ein genetischer Zufall. Die Chance, so auf die Welt zu kommen, hatte ihr Vater ihr irgendwann begeistert erklärt, lagen bei eins zu einer Million. ...Scheiß Genetik, dachte sie. Scheiß Sommer!» Gudruns persönliche Situation, die vor einem halben Jahr mit ihrer geschiedenen Mutter aus einer westdeutschen Kleinstadt in den Ostteil Berlin gezogen ist und die nicht nur unter der Fremdheit der Umgebung leidet, wo sie graubraune Häuser mit zerbröckelnden Fassaden begrüßt hatten, wo absturzgefährdete Balkons drohend über den Gehsteigen hängen und hier und da ein ausgeschlachteter Trabi zwischen den geparkten, auf Hochglanz polierten Wagen steht, die in dichten Reihen die Straßenränder säumen, charakterisiert der folgende Satz: «Stell dir vor, dein gewohntes Leben wird von heute auf morgen umgekrempelt, ohne dass du gefragt wirst, ob dir das recht ist. So fühlen sich die Leute hier.» Noch ohne Freunde versucht sie es zu verdrängen, dass sie sich sehr nach ihrem Vater sehnt, der den Kontakt mit ihr vermeidet und ihre Briefe nicht beantwortet. Mit ihrer Gefühlslage macht Steinhöfel gleichzeitig auf die stattgefundenen Veränderungen von Millionen während der Wendezeit aufmerksam: «Grau, grau, grau... sie konnte sich nicht vorstellen, wie das Leben für die Bewohner im Osten gewesen war, bevor mit den ersten Läden und Geschäften etwas Farbe in ihren Alltag eingedrungen war. Guddie denkt dabei an ihr eigenes Leben, das ebenfalls umgekrempelt worden war, ohne dass jemand sie danach gefragt hatte.»

Olaf ist der verschlossenste in der Gruppe, klaut in Warenhäusern aus innerem Zwang, verschenkt jedoch seine Beute, spricht nicht gern über seine Familie, und findet in Guddie seine erste Liebe und eine vertrauensvolle Gesprächspartnerin. Alle drei werden richtige Freunde und begreifen ihre familiäre Ähnlichkeit. Guddie fühlt sich endlich in Berlin wohl, Olaf braucht keine Angst mehr vor Entdeckungen zu haben, die drei überwinden auch ihre Vorurteile gegenüber Bernd Wörlitzer und im Gegensatz zu reinen Aktionshelden sind sie gleichzeitig Träger von Botschaften: «Männer, die sich küssen, sind mir lieber als Männer, die andere entführen, betäuben und womöglich umbringen», ist Guddies Kommentar auf Olafs anfängliches Unverständnis und seine Ablehung gegenüber dem Thema Homosexualität.

\section{Der Handlungsort: Berlin}

Zahlreiche Informationen über die Geschichte Berlins und die Museumsinsel sowie viel Lebensgefühl in der Hauptstadt sind in dieser Kriminalgeschichte verpackt. Durch anschauliche Beschreibungen von Straßen und Stadtvierteln gewährt der Autor scheinbar beiläufig aufschlussreiche Einblicke in die Berliner Stadtkultur und seine Geschichte: Berlin, der ehemalige kulturelle Nabel der Welt in den zwanziger und dreißiger Jahren und neues Modezentrum mit dem Kurfürstendamm, Berlins größter Einkaufsstraße und einstiger Promeniermeile, seinen Straßen und Gehsteigen als ein einziges Gewimmel beschrieben, eine Stadt, die nie zur Ruhe kommt mit brausendem Verkehrslärm, mitten in der Nacht lebendig und vibrierend in einem Wirbel aus bunten Lichtern, taumelnden Farben und musikalischem Lärm; Hamburger, Döner Kebaps, Böreks und belegte Fladenbrote bilden einen der Vorteile Berlins, weil man an jeder Straßenecke etwas zu essen kaufen kann, die Friedrichsbrücke, die sich bogenförmig über die grünblau funkelnde Spree spannt und zur Museumsinsel führt, das Pergamonmuseum, aus dem eins seiner größten Ausstellungstücke geraubt werden soll, der Gendarmenmarkt, Berlins schönster Platz, (so Dags), der Hausvogteiplatz, das frühere Zentrum der weltberühmten Berliner Haute Couture sind einige der Schauplätze, die den Leser mit Berlin bekannt machen, «einem Riesen, der gutmütig und bösartig, von anziehnder Schönheit und von abstoßender Hässlichkeit zugleich sein konnte.»

\section{Kreatives Schreiben: Paralleltexte erstellen}

Die Geschichte bietet aufgrund der aussparenden Informationen und unterbrechenden Erzählweise vielfältige Möglichkeiten zum handlungs- und produktionsorientierten Unterricht. Steinhöfels Erzählweise unterbricht oft, wechselt ständig die Orte und Perspektiven, überrascht durch Wiederaufgreifen fast vergessener Erzählteile, erzählt immer aus der Sicht der Kinder, nie aus der Sicht der Erwachsenen oder Verbrecher, lässt innere Stimmen der Kinder sprechen, gibt kaum Rückblicke oder Vorausdeutungen, sondern schreitet in der Zeit voran und bricht meistens an der spannendsten Stelle ab. Viele der Spannungsmomente können noch ausgeschmückt oder aus einer anderen Perspektive erzählt werden: der Kaufhausdiebstahl, die Verfolgung Gudruns auf der 
Aussichtsplattform, der Aktenkofferdiebstahl, das Fotografieren der Täter, Olafs Sturz in einen tiefen Gang, Dagmars Gefangenschaft auf einem Boot, der Sprung ins Wasser mit Romeo, der Besuch im Pergamonmuseum, die Unterhaltung mit dem Fotografen Bernd Wörlitzer und dessen Lebenspartner, die Belauschung von Griffith, dem Modezaren und seiner Geliebten usw. sind Teil einer raffiniert erzählten Kriminalgeschichte und können ausgestaltet und transformiert werden.

ein paar Ideen...

- eine Fortsetzung/einen Schluß zu einer unvollständig ausgegebenen Geschichte formulieren,

- eine Geschichte umschreiben, ein neues Ende verfassen, einen Brief an einen Protagonisten verfassen, eine Vorgeschichte zu einem Text (bzw. zu einer einzelnen Figur) schreiben, eine im Text nur angedeutete Handlung ausbauen, einen inneren Monolog, eine erlebte Rede, einen Brief oder eine Tagebuchnotiz einer Figur verfassen, in Ich-Form Figuren des Textes vorstellen («Ich heiße ...»), sich selbst in einen Text hineindichten und dazu eine Szene gestalten, eine Figur aus einer Geschichte herauslösen und in einer anderen Welt auftreten lassen (z.B. die fiktionale Figur begegnet einem in der realen Welt), einen Zeitungsreporter zu der Geschichte erfinden, eine Buchrezension schreiben, selbst eine Krimigeschichte am eigenen Wohnort schreiben, usw.

\section{Internetbasiertes Recherchieren}

Je nach der zeitlichen Disposition und den Sprachkenntnissen der Lerner kann sich die die Arbeit mit dem Buch allein auf die Lektüre und die weiterführenden kreativen Schreibaktivitäten beschränken und sicherlich zu vorzeigbaren Ergebnissen führen wie das Projektbeispiel einer Bamberger Schule illustrativ zeigt: http://www.bnv-bamberg.de/home/ba4613/flg/deutsch-interaktiv/lektueren/steinhoefel/index.htm.

Doch aufgrund der engen Verknüpfung des Handlungsortes der Geschichte mit Berlin ist es geradezu unumgänglich das Internet als Quelle für tiefere Recherchen in die Didaktisierung mitzeinzubeziehen. Damit können die Schüler selbst zu Detektiven werden und Berlin auf den Spuren der drei jugendlichen Protagonisten virtuell erforschen. Die Handlung und die Beschreibungen im Buch führen den Leser direkt auf den Ku'Damm, in den Zoo, zum Weltkugelbrunnen und zur Gedächtniskirche sowie rund um die Mueseumsinsel und natürlich ins Pergamonmuseum selbst. Dabei vergisst der Autor nicht, den Leser in die wechselvolle Geschichte Berlins einzuführen:

«Bis zum Mittelalter sah es hier noch ganz anders aus. Es gab nur den Fluss, mit den Städten Berlin auf der einen und Cölln auf der anderen Seite. Um den Schiffsverkehr besser regulieren zu können zogen die Cöllner einen Kanal durch ihre Stadt, den heutigen Kupfergraben. Er wurde später erweitert und so entstand in der Spree eine Insel. Später befand sich hier der kaiserliche Gemüsegarten».

Die folgenden Ideengrube will ein produktives Recherchieren anregen und den Umgang mit dem literarischen Text durch lernerzentrierte Rechercheaufgaben erweitern:

\section{Rechercheaufgaben}

Die Biografie des Autors, die Geschichte Berlins sowie aktuelle
Themen erforschen, kulturelle Angebote vorstellen, die Museumsinsel virtuell erforschen, eine Reise nach Berlin mit einem Kulturprogramm auf den Spuren der drei Protagonsiten planen, nach anderen Berlin-Autoren, Künstlern, Fotografen und Modemachern recherchieren, mit dem Autor per e-mail kontaktieren, ein Berlin-Quiz schreiben lassen, nach Berlin in der Literatur, in Liedern und Filmen recherchieren lassen, ...

Eine kleine Adressenauswahl über Berlin und seine Geschichte, die deutsche Mode und Literatur mag dabei nützlich sein:

http://www.museumsinsel-berlin.de

http://de.wikipedia.org/wiki/Museumsinsel_(Berlin)

http://de.wikipedia.org/wiki/Pergamonmuseum

http://commons.wikimedia.org/wiki/Pergamonmuseum

http://www.berlin-tourist-information.de

http://de.wikipedia.org/wiki/Berlin

http://www.berlin.de/berlin-im-ueberblick/geschichte/index.html http://www.literaturreisen.com/

http://www.dhm.de/lemo/

http://www.goethe.de/ins/ie/pro/projekt/DEIMODE0.HTM

http://www.goethe.de/kue/des/thm/aus/de50413.htm

http://www.goethe.de/kue/des/thm/ein/de22412.htm

Auf diese Weise lässt sich ganz schnell und mit Hilfe der Schüler eine Internet-Quiz-Ralley organisieren. Wer schon jetzt Lust auf Berlin und das Detektivspielen bekommen hat, kann sich an den folgenden Fragen versuchen:

1. Welchen Brückenschlag besonderer Art bietet das Bodemuseum?

2. Was erwartet den Besucher im Pergamonsaal?

3. Welchen Bogen spannen die Werke in der Alten Nationalgalerie?

4. Was ist die Keimzelle der Museumsinsel?

5. Welcher wichtige touristische Anlaufpunkt in Berlin gehört seit 1999 zum Weltkulturerbe der UNESCO an?

6. Welchem Gott ist der Pergamonaltar geweiht und welchen Mythos stellt er dar?

7. Wer begann mit den Ausgrabungen und wie kamen die Funde von Pergamon nach Berlin?

8. Wie heißen die acht weltberühmtesten deutschen Modedesigner?

9. Welche Lieder und Songs handeln über Berlin?

10. Welches andere Buch des gleichnamigen Autors wurde mit dem Buxterhuden Bullen ausgezeichnet?

\section{Viel Spaß und Erfolg mit dem Internet und den Beschützern der Diebe!}

Verwendete und weiterführend Weblinks zum Thema Krimi: http://de.wikipedia.org/wiki/Krimi

http://www.krimischule.de/

http://www.kriminal-literatur.de/

www.detektiv-club.de

http://www.goethe.de/ins/nl/prj/kij/unt/allg/de120099.htm

http://www.krimiautoren.at/geschichte_krimi.html

http://www.wdr.de/themen/kultur/literatur/weihnachtsraetsel/krimi.jht

ml? rubrikenstyle $=$ kultur\&rubrikenstyle $=$ kultur 